\title{
Calculated rates for the electron impact dissociation of molecular hydrogen: mixed isotopomers and scaling laws
}

\author{
Cynthia S. Trevisan and Jonathan Tennyson \\ c.trevisan@ucl.ac.uk; j.tennyson@ucl.ac.uk \\ Department of Physics and Astronomy, University College London, Gower St., \\ London WC1E 6BT, UK.
}

\begin{abstract}
Following our calculations of electron impact dissociation rates of molecular $\mathrm{H}_{2}, \mathrm{D}_{2}$ and $\mathrm{T}_{2}$ (Trevisan \& Tennyson 2002, Plasma Phys. Control. Fusion, In Press), calculations for the mixed isotopomers HD, HT and DT have been made. Total cross sections and energy differential cross sections at threshold energies as a function of vibrational states $(v)$ for HD $v=0-5$, HT $v=0-5$ and DT $v=0-7$ are calculated for the electron impact dissociation through excitation to the $\mathrm{b}^{3} \Sigma_{u}^{+}$excited electronic state, which is the dominant dissociation process at such energies. The rates of dissociation as a function of electron temperature for each state are parametrized. Coinciding with our previous results, near-threshold rates are shown to be so critically dependent on the vibrational level that dissociation from very high-lying vibrational levels must be included in calculations of the rate at local thermal equilibrium (LTE) even at low temperature. Rates for the higher vibrational levels are extrapolated following the procedure discussed in our previous publication, and are then used to calculate the LTE rate. The LTE rate is an order of magnitude greater than the $v=0$ rate. Calculations of energy differential cross sections suggest that impact dissociation of vibrationally excited molecules could be the source of low energy $\mathrm{H}$ atoms observed in tokamak plasmas. A scaling law for the electron impact dissociation cross sections of vibrationally excited $\mathrm{H}_{2}$ and its isotopomers has been derived.
\end{abstract}

\section{Introduction}

Molecular collision processes and their quantitative characteristics, such as cross sections and transition rates, are important when describing the properties and dynamics of low temperature plasmas. Particularly, modelling of cool plasmas requires the knowledge of a large number of cross sections involving electron-molecule interactions. Most information, both experimental and theoretical, is limited to the various processes involving the ground vibrational state of $\mathrm{H}_{2}$ and $\mathrm{D}_{2}$ molecules so that their use is limited to very cool plasmas in which the presence of vibrationally excited molecules can be neglected.

In many cases, however, the vibrational excitation of the molecule cannot be neglected, and cross sections involving the different vibrational states should be known 
(Capitelli \& Celiberto 1995). Indeed, subtle effects in the detailed physics can have consequences for the plasma. For example, the source of many low energy $\mathrm{H}$ atoms observed in tokamak plasmas (Mertens et al. 2001, Tanaka et al. 2000, Hey et al. 2000) remains unexplained.

Experimentally it is difficult to measure cross sections (or rates) for electron impact dissociation of a neutral species into neutral fragments. Only limited experimental data is available for this process (Corrigan 1965, Khakoo \& Segura 1994, Nishimura \& Danjo 1986, Hall \& Andrić 1984), all of it for $\mathrm{H}_{2}$ in its $v=0$ vibrational ground state. However, our previous calculations (Trevisan \& Tennyson 2002) have shown that the rate of near threshold electron impact dissociation is strongly dependent on the initial vibrational state of $\mathrm{H}_{2}, \mathrm{D}_{2}$ and $\mathrm{T}_{2}$. The information about cross sections involving vibrationally excited states comes from theoretical considerations.

An extensive database of cross sections for inelastic electron-impact processes of vibrationally excited hydrogen and its isotopes has been compiled by Celiberto et al (2001). Cross sections reported for the singlet-triplet transition $\mathrm{X}^{1} \Sigma_{g}^{+} \rightarrow \mathrm{b}^{3} \Sigma_{u}^{+}$were obtained using a semiclassical method (Celiberto et al. 1989, Bauer \& Bartky 1965) for $\mathrm{H}_{2}$ and $\mathrm{D}_{2}$. This method should give satisfactory results at high impact electron energies and hence temperatures, but is not reliable in the near-threshold (low temperature) regime were subtle quantum effects must be correctly modelled. Comparisons between these results and our calculations can be found in Trevisan \& Tennyson (2002). No data involving this transition is reported for isotopomers other than $\mathrm{H}_{2}$ and $\mathrm{D}_{2}$.

Celiberto et al (2001) also give analytical fits of the numerical cross section data and provide scaling relationships for the electronic excitation $\mathrm{X}^{1} \Sigma_{g}^{+} \rightarrow \mathrm{B}^{1} \Sigma_{u}^{+}$and $\mathrm{X}^{1} \Sigma_{g}^{+} \rightarrow$ $\mathrm{C}^{1} \Pi_{u}$ transitions, and the processes of dissociative ionization and dissociative electron attachment. They did not extend their work onto the singlet-triplet transition under study here nor to the electron impact dissociation process.

Following our previous work (Trevisan \& Tennyson 2002), we present the total cross sections and rates for electron impact dissociation of HD, HT and DT as a function of the vibrational states. We use the adiabatic nuclei approximation and the energy balance model of Stibbe and Tennyson (1998a) to obtain the appropriate energy-dependent Tmatrices. This model assumes that the dissociation of $\mathrm{H}_{2}$ for energies below around $12 \mathrm{eV}$ proceeds exclusively via electronic excitation to the first excited state of $\mathrm{H}_{2}$ :

$$
e+H_{2}\left(X^{1} \Sigma_{g}^{+}\right) \rightarrow H_{2}^{*}\left(b^{3} \Sigma_{u}^{+}\right)+e \rightarrow H(1 s)+H(1 s)+e .
$$

The formal expressions needed for such calculations were derived previously (Trevisan \& Tennyson 2001). Here we extend these calculations to the mixed isotopomers. Specifically, we consider $v=0-5 \mathrm{HD}, v=0-5 \mathrm{HT}$ and $v=0-7$ DT vibrational states, and produce rates for such vibrational states. The extrapolation procedure developed in Trevisan \& Tennyson (2001) to approximate the rates for all of the higher vibrational levels is used to calculate the local thermal equilibrium (LTE) rates. We also derive a scaling law for the electron impact dissociation cross sections of $\mathrm{H}_{2}$ and its isotopomers for the above mentioned lower vibrational states. This scaling law 
has an explicit dependence on the reduced mass of the isotopomer and the vibrational state considered.

\section{Method and Results}

The expression used for the calculation of differential and total cross sections, developed in our previous study (Trevisan \& Tennyson 2001) and generalized to any molecule, is

$$
\frac{\mathrm{d} \sigma}{\mathrm{d} E_{k e}}=\frac{\mu}{2 \pi^{3} m_{e}} \frac{E_{k e}}{E_{\text {in }}} \sum_{\Lambda \nu l_{i} l_{j}}\left|T_{\bar{i} \bar{l}_{i} \bar{j} \bar{l}_{j}}^{\Lambda \nu}\left(E_{\text {in }}, E_{\text {out }}\right)\right|^{2}
$$

where $\mu$ represents the reduced mass of the molecule, $m_{e}$ is the mass of the electron, $E_{i n}$ the incoming electron energy, $E_{k e}$ the energy of the dissociating atoms and $E_{\text {out }}$ the energy of the scattered electron. $T T_{\bar{i} l_{i} j \bar{l}_{j}}^{\Lambda \nu}\left(E_{i n}, E_{\text {out }}\right)$ are the transition matrix elements, where the indices $\mathrm{i}, \mathrm{j}$ label the initial and final electronic states of the molecule and represent all quantum numbers needed to specify the electronic state $i, j$ of the molecule, respectively. $\bar{i}, \bar{j}$ stand for $\left(\Lambda_{i}, S_{i}, \nu_{i}\right)$ and $\left(\Lambda_{j}, S_{j}, \nu_{j}\right)$, and $\bar{l}_{i}, \bar{l}_{j}$ run over values satisfying $(-1)^{\bar{l}_{i}} \nu_{i}=\nu ;(-1)^{\bar{l}_{j}} \nu_{j}=\nu ; \Lambda$ is the projection of the total electronic angular momentum on the internuclear axis; $\mathrm{S}$ represents the total spin and $\nu$ labels the gerade or ungerade symmetry in homonuclear molecules.

The mass dependence in eq. (2) comes from considering the density of final states for a three body problem in the output channel. Because its derivation is independent of any particular electronic state, we expect the same scaling of cross sections for all dissociation processes as a function of the isotopic reduced mass.

Mixed isotopomers have lower symmetry than $\mathrm{H}_{2}$ and their centre-of-mass does not coincide with the geometric centre of the molecule. These changes could affect the electron scattering T-matrices and cross sections. We therefore performed a series of $\mathrm{C}_{\infty v}$ symmetry R-matrix calculations centred on the HT centre-of-mass. These gave results that differed negligibly from the corresponding calculations on $\mathrm{H}_{2}$. We therefore concluded that the T-matrices originally computed for $\mathrm{H}_{2}$ could be used for the mixed isotopomers without significant loss of accuracy.

The T-matrices used were originally calculated for an investigation of $\mathrm{H}_{2}^{-}$resonances as a function of the internuclear distance R (Stibbe and Tennyson 1997a, 1997b, 1998b). The generalization of the adiabatic nuclei approximation to electron impact dissociation is discussed in detail by Stibbe and Tennyson (1998a). Such geometry-dependent, off-shell fixed-nuclei T-matrices were averaged between nuclear wave functions for the vibrational states and nuclear motion continuum functions with appropriate energy to represent the dissociating state.

Nuclear continuum wavefunctions, $\Xi_{c}\left(E_{k e}, R\right)$, were calculated by directly solving the Schrödinger equation for the dissociating potential, and energy normalized. The potential of Kolos and Wolniewicz (1965) was used in place of that implicit in the T-matrix calculations, which is $(\sim 0.1 \mathrm{eV})$ less accurate. The initial vibrational 
wavefunctions, $\Xi_{v}(R)$, and energies were found with the program LEVEL (LeRoy 1996) also using the former potential.

Total cross sections are given by

$$
\sigma\left(E_{i n}\right)=\int_{0}^{E_{i n}-D_{v}} \frac{\mathrm{d} \sigma}{\mathrm{d} E_{k e}} d E_{k e}
$$

with $D_{v}$ the vibrational state-dependent dissociation energy. No allowance is made for the possible effect of rotational motion of the diatoms.

Figures 1 to 6 show the dissociation cross sections for $\mathrm{H}_{2}(v), \mathrm{HD}(v), \mathrm{HT}(v), \mathrm{D}_{2}(v)$, $\mathrm{DT}(v)$ and $\mathrm{T}_{2}(v)$ as a function of incoming electron energy. Although cross sections are shown up to values of $E_{\text {in }}$ that go beyond $12 \mathrm{eV}$, our calculations are expected to be accurate only up to around that value. Above that energy, the molecule can be excited to higher states from which it can dissociate directly or cascade down to the repulsive state. Another possibility above $12 \mathrm{eV}$ is the excitation of an $\mathrm{H}_{2}$ resonance state which can then decay into the $\mathrm{b}^{3} \Sigma_{u}^{+}$state. The adiabatic model we use is not valid for these long-lived resonances and the additional dissociation pathways above 12 $\mathrm{eV}$ are not included in our model.

Because of the finite range of internuclear separations $\left(\mathrm{R}=0.8-4.0 a_{o}\right)$ for which T-matrices are available, full calculations are only possible for $v \leq 4$ in $\mathrm{H}_{2}(v), v \leq 5$ in $\operatorname{HD}(v), v \leq 5$ in $\mathrm{HT}(v), v \leq 6$ in $\mathrm{D}_{2}(v), v \leq 7$ in $\mathrm{DT}(v)$ and $v \leq 7$ in $\mathrm{T}_{2}(v)$. In order to obtain accurate cross sections for higher vibrational states, T-matrices must be calculated over a much wider range of bond length. Our present calculation cannot be extended easily to provide these T-matrices because of basis set limitations and because, at long bond length, the target electronic wave function will leak out of the R-matrix boundary.

Figures 1 to 6 also illustrate the scaled dissociation cross sections for the different initial vibrational states of $\mathrm{H}_{2}(v), \mathrm{HD}(v), \mathrm{HT}(v), \mathrm{D}_{2}(v), \mathrm{DT}(v)$ and $\mathrm{T}_{2}(v)$. In deriving a scaling law for these species a number of properties have been used:

- The threshold energy decrease with increasing $v$ is related to the rapid decrease of the vertical target excitation (or fixed nuclei) energy at larger internuclear separations. This threshold is largely determined by the vertical excitation at the vibrationally-dependent outer turning point of the potential. Such variation has a significant role when including nuclear motion. At the same time, low energy dissociation is predicted to occur predominantly from the outer classical turning point, thus determining the energy shifts, $\Delta E_{\mu}(v)$, to use for the different vibrational states in our scaling law. These values arise from the energy difference between the $b^{3} \Sigma_{u}^{+}$and the $X^{1} \Sigma_{g}^{+}$potential curves of $\mathrm{H}_{2}(v)$ (vertical target excitation energy) at the outer classical turning point for each vibrational level.

- At high energy, the isotopomer dependence of the cross section is simply proportional to the isotopomer's reduced mass, $\mu$.

- Cross sections decrease as the vibrational state increases. 
- The tanh (or "switch") function satisfactorily reproduces the shape of the $\mathrm{H}_{2}(0)$ cross section curve at the relevant incoming electron energies.

Taking the above items into account, a simple scaling law applicable to every isotopomer was derived from parametrizing the following expression to the $v=0$ vibrational state for $\mathrm{H}_{2}(v)$ :

$$
\sigma=2 \mu\left(1-\frac{v}{v_{\text {max }}^{2}}\right) a_{0} \tanh \left(a_{1}\left(E_{\text {in }}+\Delta E_{\mu}(v)\right)+a_{2}\right)+a_{3}
$$

where $\mu$ is the reduced mass of the isotopomer in atomic units, $v$ the vibrational state considered, $v_{\max }$ the highest vibrational state for which we can perform full calculations in each case and $a_{0}$ to $a_{3}$ are coefficients that arise from the parametrization. As before, $E_{i n}$ is the energy of the incoming electron in $\mathrm{eV} . \Delta E_{\mu}(v)$ is the shift in the threshold energy for each vibrational state and each isotopomer, also in $\mathrm{eV}$. The parametrization coefficients, and the energy shifts and $v_{\max }$ values for the different isotopomers are given in Table 1 and Table 2, respectively.

The tanh function in eq.(4) tends to unity at high values of $E_{\text {in }}$ and to -1 at small $E_{\text {in }}$ values. To follow the $\mathrm{H}_{2}(0)$ cross section curve satisfactorily above the threshold region, it needs to be shifted upwards, which is done by the parameter $a_{3}$. The multiplying factor, $a_{0}$, of tanh in eq.(4) is negative, and adjusts the point of inflection in the curve to the correct energy. This means, however, that for sub-threshold values of $E_{i n}$, eq.(4) will give negative cross sections. In this case, the cross section should simply be set to zero, i.e. $\sigma=\max (\sigma, 0)$. At high $E_{\text {in }}$ values, this scaling law gives flat curves.

\begin{tabular}{cccc}
\hline$a_{0}$ & $a_{1}$ & $a_{2}$ & $a_{3}$ \\
\hline-0.9000 & -0.9296 & 17.6080 & 0.8504 \\
\hline
\end{tabular}

Table 1. Parameters for scaled electron impact dissociation cross sections. Cross sections are in $a_{0}^{2}$ for energies in $\mathrm{eV}$ and reduced mass in atomic units.

Table 3 gives the mean values of the dissociation cross section curves calculated fully and the standard deviations of the difference between the calculated dissociation cross sections and those obtained by our scaling law for each of the vibrational states involved. It shows that the typical error is about $15 \%$, similar to that achieved by Celiberto et al. (1996). As can be observed in figures 1 to 6 , calculated cross sections will be described rather satisfactorily at energies up to aproximately $2 \mathrm{eV}$ above the threshold value of each vibrational state. Extrapolations to regions above $E_{i n}=12 \mathrm{eV}$ and to vibrational states with $v>v_{\max }$ can be performed, but should be handled with caution.

Energy differential cross sections contour plots, where the energy of the dissociating atoms, $E_{k e}$ is plotted against the electron impact energy, $E_{i n}$, have also been calculated for HD, HT and DT, for each of the initial bound vibrational states for which our T-matrices were able to support full calculations. These are qualitatively similar to 


\begin{tabular}{ccccccc}
\hline$v$ & $\mathrm{H}_{2}$ & $\mathrm{HD}$ & $\mathrm{HT}$ & $\mathrm{D}_{2}$ & $\mathrm{DT}$ & $\mathrm{T}_{2}$ \\
\hline 0 & 8.74 & 8.87 & 8.87 & 9.01 & 9.08 & 9.22 \\
1 & 7.07 & 7.32 & 7.50 & 7.69 & 7.82 & 8.00 \\
2 & 5.93 & 6.26 & 6.43 & 6.78 & 6.89 & 7.14 \\
3 & 4.92 & 5.36 & 5.51 & 5.93 & 6.15 & 6.43 \\
4 & 4.08 & 4.55 & 4.74 & 5.21 & 5.46 & 5.77 \\
5 & - & 3.88 & 4.12 & 4.55 & 4.87 & 5.21 \\
6 & - & - & - & 4.00 & 4.33 & 4.69 \\
7 & - & - & - & - & 3.80 & 4.25 \\
$v_{\max }$ & 4 & 5 & 5 & 6 & 7 & 7 \\
\hline
\end{tabular}

Table 2. Energy shifts, $\Delta E_{\mu}(v)$, for each vibrational state and each istopomer in $\mathrm{eV}$, and highest vibrational state, $v_{\max }$, for which full cross section calculations can be performed for each isotopomer.

\begin{tabular}{cccccccc}
\hline & $v$ & $\mathrm{H}_{2}$ & $\mathrm{HD}$ & $\mathrm{HT}$ & $\mathrm{D}_{2}$ & $\mathrm{DT}$ & $\mathrm{T}_{2}$ \\
\hline Mean & 0 & 0.77 & 1.02 & 1.14 & 1.52 & 1.82 & 2.26 \\
Std. dev. & 0 & 0.08 & 0.11 & 0.13 & 0.18 & 0.22 & 0.34 \\
Mean & 1 & 0.85 & 1.11 & 1.24 & 1.64 & 1.95 & 2.42 \\
Std. dev. & 1 & 0.12 & 0.17 & 0.19 & 0.25 & 0.30 & 0.36 \\
Mean & 2 & 0.91 & 1.19 & 1.32 & 1.73 & 2.06 & 2.54 \\
Std. dev. & 2 & 0.10 & 0.16 & 0.18 & 0.26 & 0.32 & 0.39 \\
Mean & 3 & 0.96 & 1.25 & 1.40 & 1.82 & 2.15 & 2.65 \\
Std. dev. & 3 & 0.09 & 0.14 & 0.16 & 0.25 & 0.32 & 0.40 \\
Mean & 4 & 1.01 & 1.31 & 1.46 & 1.90 & 2.24 & 2.75 \\
Std. dev. & 4 & 0.09 & 0.13 & 0.14 & 0.23 & 0.30 & 0.37 \\
Mean & 5 & - & 1.37 & 1.52 & 1.97 & 2.32 & 2.84 \\
Std. dev. & 5 & - & 0.13 & 0.14 & 0.21 & 0.29 & 0.35 \\
Mean & 6 & - & - & - & 1.81 & 2.40 & 2.93 \\
Std. dev. & 6 & - & - & - & 0.25 & 0.27 & 0.33 \\
Mean & 7 & - & - & - & - & 2.47 & 3.02 \\
Std. dev. & 7 & - & - & - & - & 0.26 & 0.32 \\
\hline
\end{tabular}

Table 3. Mean value of fully calculated dissociation cross sections and standard deviations of the difference between the calculated dissociation cross sections and those obtained by our scaling law for each vibrational state and each istopomer. Cross sections are in $a_{0}^{2}$

those of $\mathrm{H}_{2}, \mathrm{D}_{2}$ and $\mathrm{T}_{2}$ presented previously (Trevisan \& Tennyson 2002) so they are not included is this paper but are available upon request. Again, they support the predictions that low energy dissociation is predominantly from the outer turning point of the $\mathrm{H}_{2}$ ground state potential and could explain the presence of cold atoms which 
originate from electron impact dissociation from higher vibrational states.

The rate of electron impact dissociation of the diatom in vibrational state $v$ as a function of electron temperature $T_{e}$ is calculated from the relevant cross sections using the standard formula

$$
q_{v}\left(T_{e}\right)=\frac{8 \pi}{m_{e}^{1 / 2}}\left(\frac{1}{2 \pi k T_{e}}\right)^{3 / 2} \int_{0}^{\infty} \sigma_{v}(E) e^{-E / k T_{e}} E d E
$$

where $\mathrm{k}$ is the Boltzmann constant and $m_{e}$ is the electron mass. For the temperatures under consideration here (up to $15,000 \mathrm{~K}$ ), the threshold region of dissociation provides by far the larger contribution to the total rate. The upper limit for the integral in eq. (5) of $15 \mathrm{eV}$ should give an accurate rate at temperatures below 15,000 K.

Following the extrapolation procedure discussed in detail in Trevisan \& Tennyson (2002), we extrapolated thermal rates for the higher vibrational levels $6 \leq v \leq 16$ in $\mathrm{HD}, 6 \leq v \leq 17$ in HT and $8 \leq v \leq 22$ in DT. Figures 7 to 9 show both the calculated (full curves) and extrapolated (broken curves) rates and the local thermal equilibrium rate (chain curve). As with our previous calculations for $\mathrm{H}_{2}, \mathrm{D}_{2}$, and $\mathrm{T}_{2}$, we found the rate dependence on the initial vibrational level to be strong, with the LTE rate for each isotopomer being an order of magnitude greater than the $v=0$ rate. This is significant when considering total rates for a particular distribution of molecules among its vibrational levels. The $v=0$ value for the rate is often used to approximate the total dissociation rate. However, the $v$ dependence is so critical that this is unlikely to be a good approximation in many situations.

The rates $\left(\right.$ in $\mathrm{cm}^{3} \mathrm{~s}^{-1}$ ) are parametrized to the form

$$
q(T)=a T^{b} \exp \left(-\frac{c}{T}\right) \times 10^{-9}
$$

and the parameters are given in Table 4. Although we do not have a formal statistical deviation for our fits, the difference tolerance is guaranteed to be below $0.01 \%$.

Table 4. Parameters for the fit formula of the electron impact dissociation rates in $\mathrm{cm}^{3} \mathrm{~s}^{-1}$ for $v \leq 5$ in $\operatorname{HD}(v), v \leq 5$ in $\operatorname{HT}(v)$ and $v \leq 7$ in $\mathrm{DT}(v)$ assuming a thermal electron distribution, and the local thermal equilibrium (LTE) rate for each diatom. $T_{\min }$ is the cutoff temperature below each rate is under $10^{-14} \mathrm{~cm}^{3} \mathrm{~s}^{-1}$ and effectively zero. 


\begin{tabular}{lcccc}
\hline & $T_{\min }(\mathrm{K})$ & $\mathrm{a}$ & $\mathrm{b}$ & $\mathrm{c}$ \\
\hline HD $v=0$ & 6150 & 5.0857 & 0.128471 & 103258.0 \\
HD $v=1$ & 5300 & 7.8899 & 0.068619 & 88400.5 \\
HD $v=2$ & 4600 & 4.8774 & 0.103393 & 76138.1 \\
HD $v=3$ & 4000 & 3.3749 & 0.129047 & 65959.1 \\
HD $v=4$ & 3450 & 1.2851 & 0.212397 & 56361.5 \\
HD $v=5$ & 3000 & 0.5611 & 0.283211 & 47950.9 \\
HD LTE & 4050 & 1.0422 & 0.217739 & 53070.7 \\
HT $v=0$ & 6150 & 5.9524 & 0.126166 & 103919.0 \\
HT $v=1$ & 5300 & 5.5017 & 0.115624 & 88952.2 \\
HT $v=2$ & 4650 & 4.2107 & 0.130195 & 77333.6 \\
HT $v=3$ & 4100 & 2.7580 & 0.161464 & 67385.2 \\
HT $v=4$ & 3550 & 1.5692 & 0.206774 & 58505.0 \\
HT $v=5$ & 3100 & 0.7659 & 0.267281 & 50400.6 \\
HT LTE & 4000 & 2.7109 & 0.136288 & 53944.4 \\
DT $v=0$ & 6150 & 8.7605 & 0.139531 & 106038.0 \\
DT $v=1$ & 5400 & 8.0730 & 0.129440 & 93150.8 \\
DT $v=2$ & 4850 & 8.6245 & 0.112493 & 83372.2 \\
DT $v=3$ & 4400 & 8.1416 & 0.109786 & 75000.5 \\
DT $v=4$ & 3950 & 5.1450 & 0.146258 & 67155.8 \\
DT $v=5$ & 3550 & 3.2584 & 0.182999 & 60080.5 \\
DT $v=6$ & 3200 & 1.4341 & 0.254987 & 53198.0 \\
DT $v=7$ & 2850 & 0.8614 & 0.297455 & 47206.6 \\
DT LTE & 3900 & 3.0924 & 0.169113 & 53475.2 \\
\hline
\end{tabular}

The Franck-Condon cross sections were calculated using the $\mathrm{H}_{2}$ potential curve of Kolos and Wolniewicz (1965) for all of the vibrational states. Rates were calculated using eq. (5) with cross sections obtained from our full T-matrices calculations and from the Franck-Condon factor cross sections for those states for which full calculations are not possible; they are illustrated in figures 7 to 9 . These rates were used to find the LTE rate for each molecule. The LTE rates are also illustrated in figures 7 to 9 and parametrized in Table 4.

Figure 10 compares the LTE rates of the isotopomers studied. Although less noticeable than with the dissociation cross sections, the rates are sensitive to the mass of the particular molecule analyzed, with rates increasing with the reduced mass of the diatom.

\section{Conclusions}

There is very limited data available for the low energy electron impact dissociation from vibrationally excited states of $\mathrm{H}_{2}$ and $\mathrm{D}_{2}$, and none for $\mathrm{HD}, \mathrm{HT}$, DT nor $\mathrm{T}_{2}$. Following 
our previous work on $\mathrm{H}_{2}(v), \mathrm{D}_{2}(v)$ and $\mathrm{T}_{2}(v)$, we have calculated accurately the rate of dissociation for $v \leq 5$ in $\operatorname{HD}(v), v \leq 5$ in $\operatorname{HT}(v)$ and $v \leq 7$ in $\mathrm{DT}(v)$ and found that the rate increases so dramatically with $v$ that all of the initial vibrational levels must be included in a calculation of the rate at local thermal equilibrium.

From the results of the vibrational states for which we can perform full calculations, we adapt the procedure discussed in (Trevisan \& Tennyson 2002) to estimate the rates of dissociation from the remaining vibrational levels, $6 \leq v \leq 16$ in $\operatorname{HD}(v), 6 \leq v \leq 17$ in $\operatorname{HT}(v)$ and $8 \leq v \leq 22$ in $\mathrm{DT}(v)$. The LTE rates for each molecule were calculated from these rates and found to be at least an order of magnitude greater than the $v=0$ rates. The fact that the LTE rates are so much higher than the $v=0$ rates is important given that, with the lack of any alternative, the $v=0$ rates are likely to be used as approximations to the LTE rates.

The cross sections depend on the isotope's mass and, hence, our calculated rates will also be sensitive to the specific diatom considered. Nevertheless, because of the Boltzmann distribution assumed among the vibrational levels, such mass effect will be less noticeable when calculating dissociation rates than in the actual dissociation cross sections.

\section{Acknowledgements}

We wish to thank Jimena Gorfinkiel and Thornton Greenland for useful discussions and guidance.

Bauer E \& Bartky C D 1965 J. Chem. Phys. 43, 2466.

Capitelli M \& Celiberto R 1995 Atomic and Molecular Processes in Fusion Edge Plasmas R. K. Janev, Plenum Press New York and London.

Celiberto R, Cacciatore M, Capitelli M \& Gorse C 1989 Chem. Phys. 133, 355.

Celiberto R, Capitelli M \& Janev R K 1996 Chem. Phys. Lett. 256, 575-580.

Celiberto R, Janev R K, Laricchiuta A, Capitelli M, Wadehra J M\& D. Atems E 2001 At. Data and Nucl. Data Tables 77, 161-213.

Corrigan S J B 1965 J. Chem. Phys. 43, 4381-4386.

Hall R I \& Andrić L 1984 J. Phys. B: At. Mol. Phys. 17, 3815-3825.

Hey J D, Chu C C \& Hintz E 2000 Contrib. Plasma Phys. 40, 1-2, 9-22.

Khakoo M A \& Segura J 1994 J. Phys. B: At. Mol. Opt. Phys. 27, 2355-2368.

Kolos W and Wolniewicz L 1965 J. Chem. Phys. 432429

LeRoy R 1996 University of Waterloo Chemical Physics Research Report CP-555R, 1.

Mertens P, Brezinsek S, Greenland P T, Hey J D, Pospieszczyk A, Reiter D, Samm U, Schweer Sergienko

G \& Vietzke E 2001 Plasma Phys. Control. Fusion 43 A349-A373

Nishimura H \& Danjo A 1986 J. Phys. Soc. Japan 55,9, 3031-3036.

Stibbe D. T \& Tennyson J 1997 a Phys. Rev. Lett. 794116

Stibbe D. T \& Tennyson J 1997b J. Phys. B: At. Mol. Opt. Phys. 30 L301

Stibbe D. T \& Tennyson J 1998a New J. Phys. 1 2.1-2.9

Stibbe D. T \& Tennyson J 1998b J. Phys. B: At. Mol. Opt. Phys. 31815

Tanaka S, Xiao B, Kazuki K \& Morita M 2000 Plasma Phys. Control. Fusion 42, 1091-1103.

Trevisan C S \& Tennyson J 2001 J. Phys. B: At. Mol. Opt. Phys. 34, 2935-2949. 
Trevisan C S \& Tennyson J 2002 Plasma Phys. Control. Fusion In Press (provisionally scheduled for July 2002) 


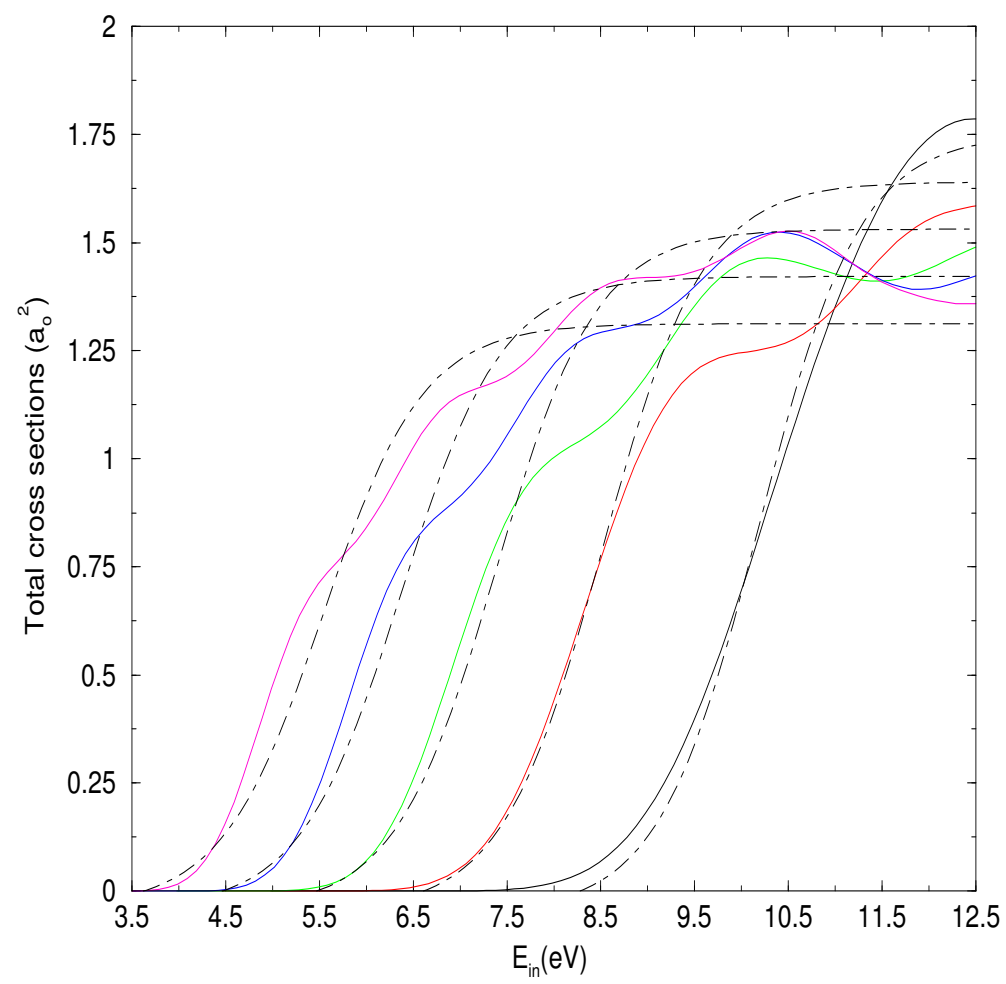

Figure 1. Electron impact dissociation cross sections for $\mathrm{H}_{2}$ as a function of the incoming electron energy, $E_{i n}$ and initial vibrational level $v$. Full curves from right to left are the cross sections obtained from our full T-matrices calculations. Chained curves from right to left illustrate the curves obtained by applying our scaling law to each of the vibrational states described. 


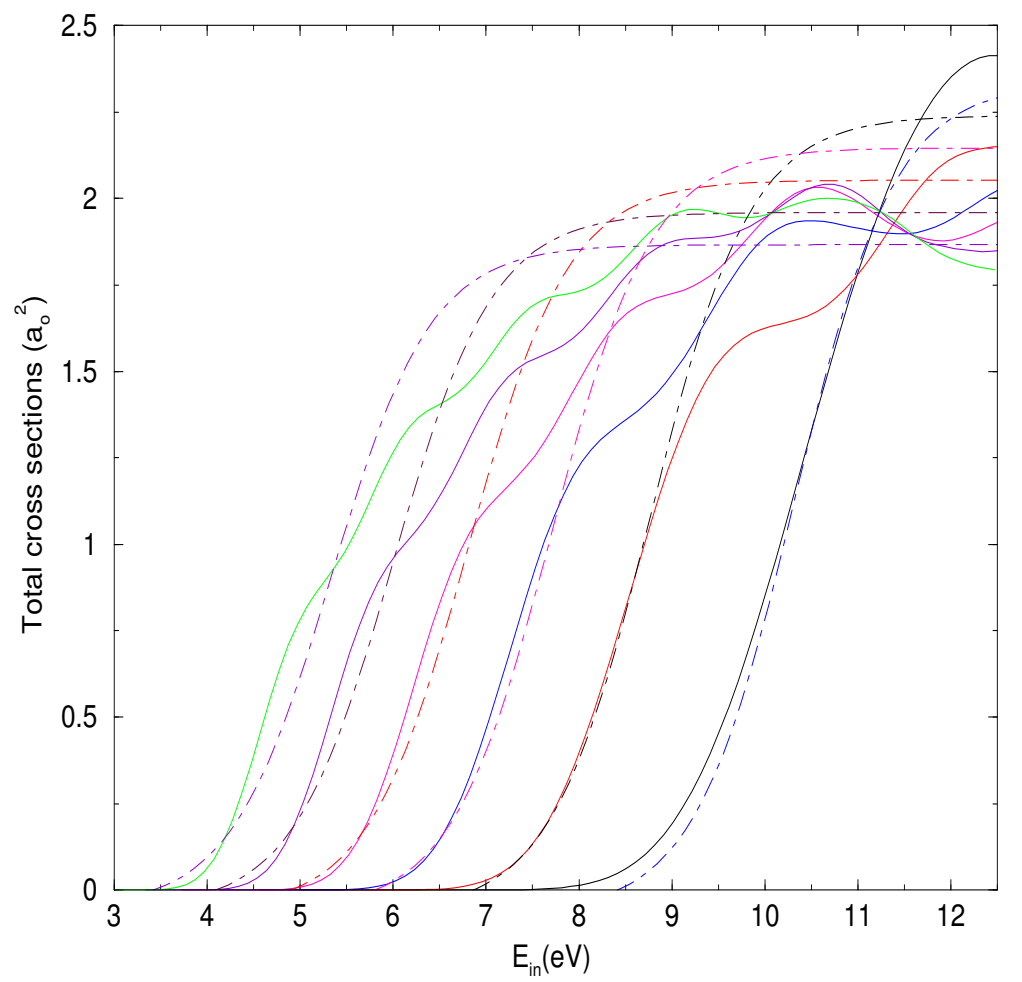

Figure 2. Electron impact dissociation cross sections for HD as a function of the incoming electron energy, $E_{i n}$ and initial vibrational level $v$. Full curves from right to left are the cross sections obtained from our full T-matrices calculations. Chained curves from right to left illustrate the curves obtained by applying our scaling law to each of the vibrational states described. 


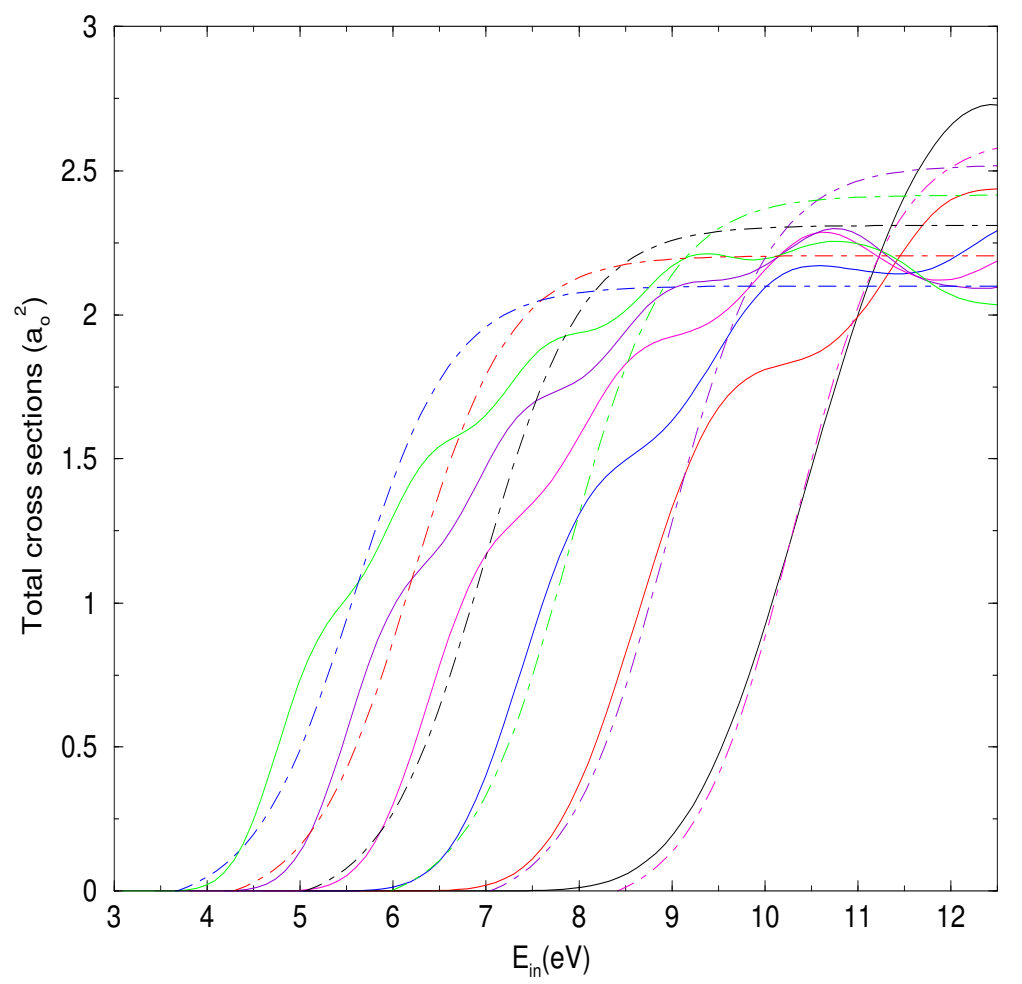

Figure 3. Electron impact dissociation cross sections for $\mathrm{HT}$ as a function of the incoming electron energy, $E_{i n}$ and initial vibrational level $v$. Full curves from right to left are the cross sections obtained from our full T-matrices calculations. Chained curves from right to left illustrate the curves obtained by applying our scaling law to each of the vibrational states described. 


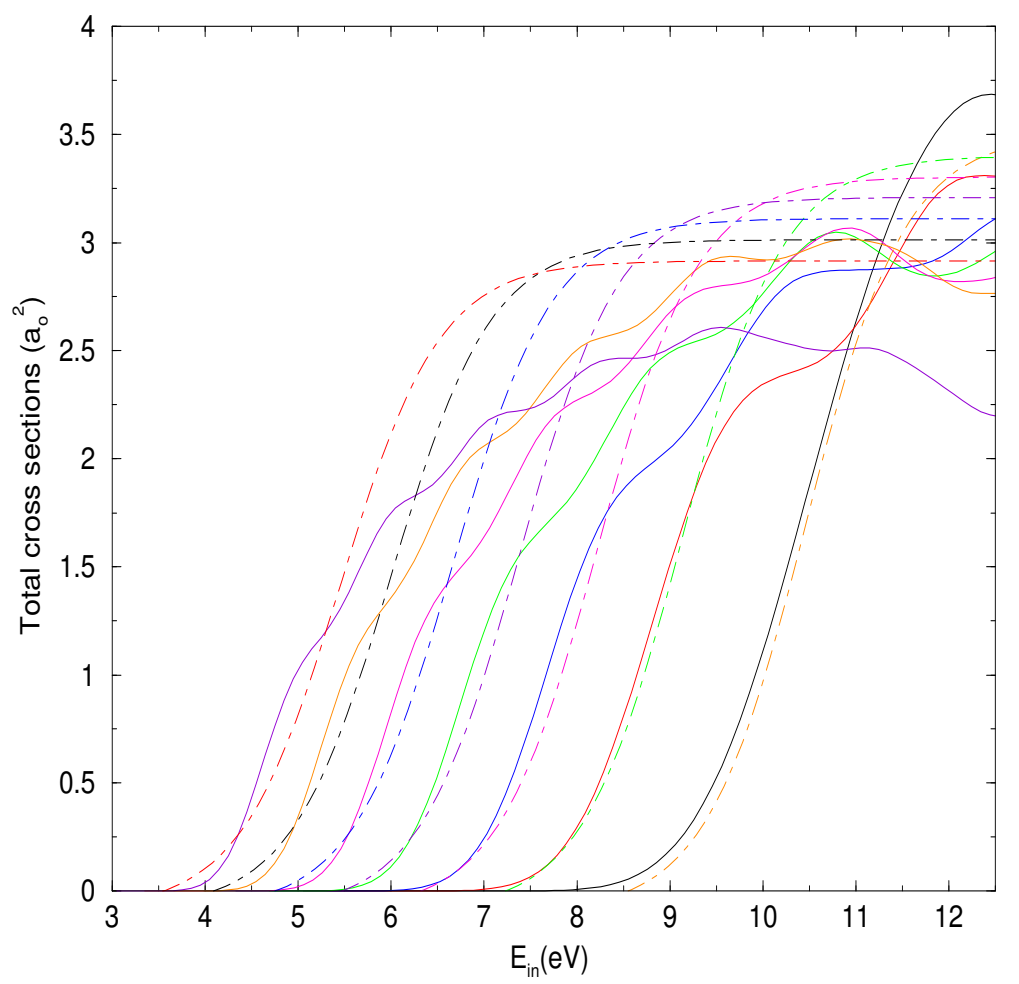

Figure 4. Electron impact dissociation cross sections for $D_{2}$ as a function of the incoming electron energy, $E_{i n}$ and initial vibrational level $v$. Full curves from right to left are the cross sections obtained from our full T-matrices calculations. Chained curves from right to left illustrate the curves obtained by applying our scaling law to each of the vibrational states described. 


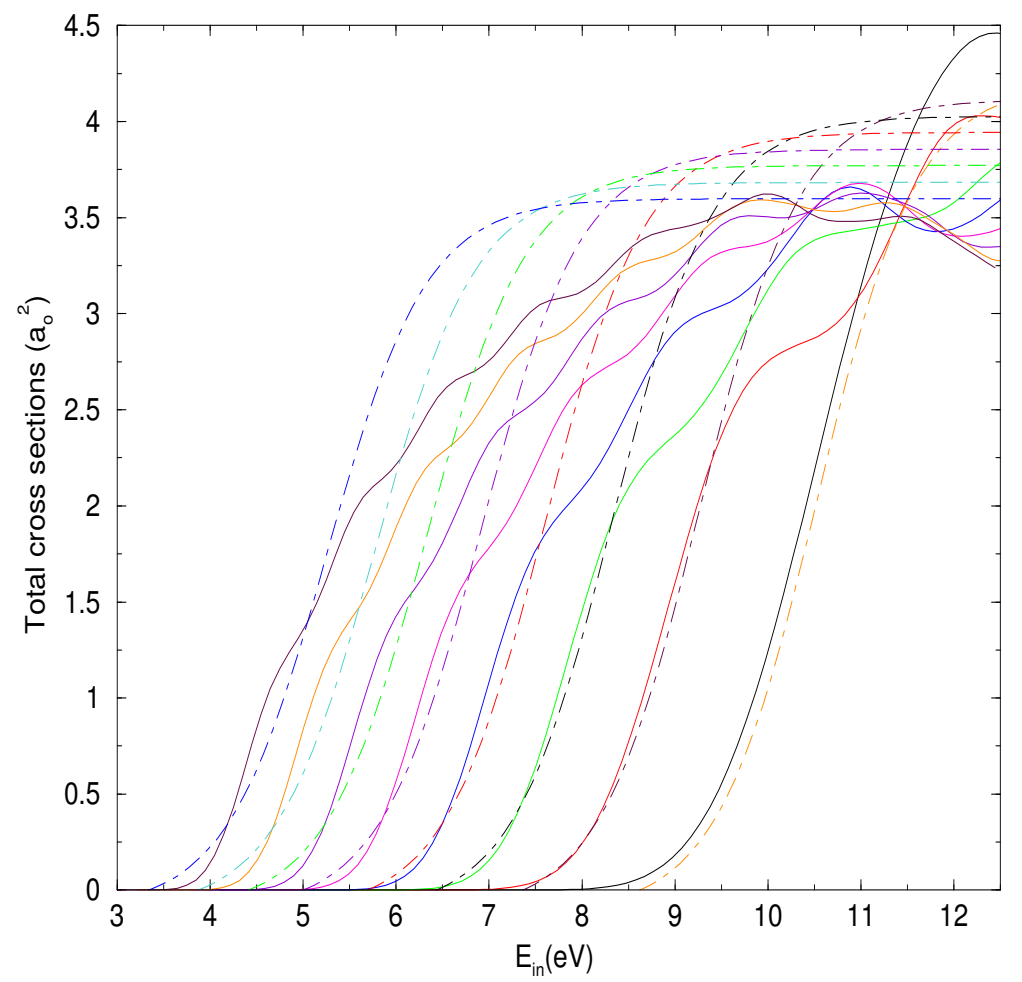

Figure 5. Electron impact dissociation cross sections for DT as a function of the incoming electron energy, $E_{i n}$ and initial vibrational level $v$. Full curves from right to left are the cross sections obtained from our full T-matrices calculations. Chained curves from right to left illustrate the curves obtained by applying our scaling law to each of the vibrational states described. 


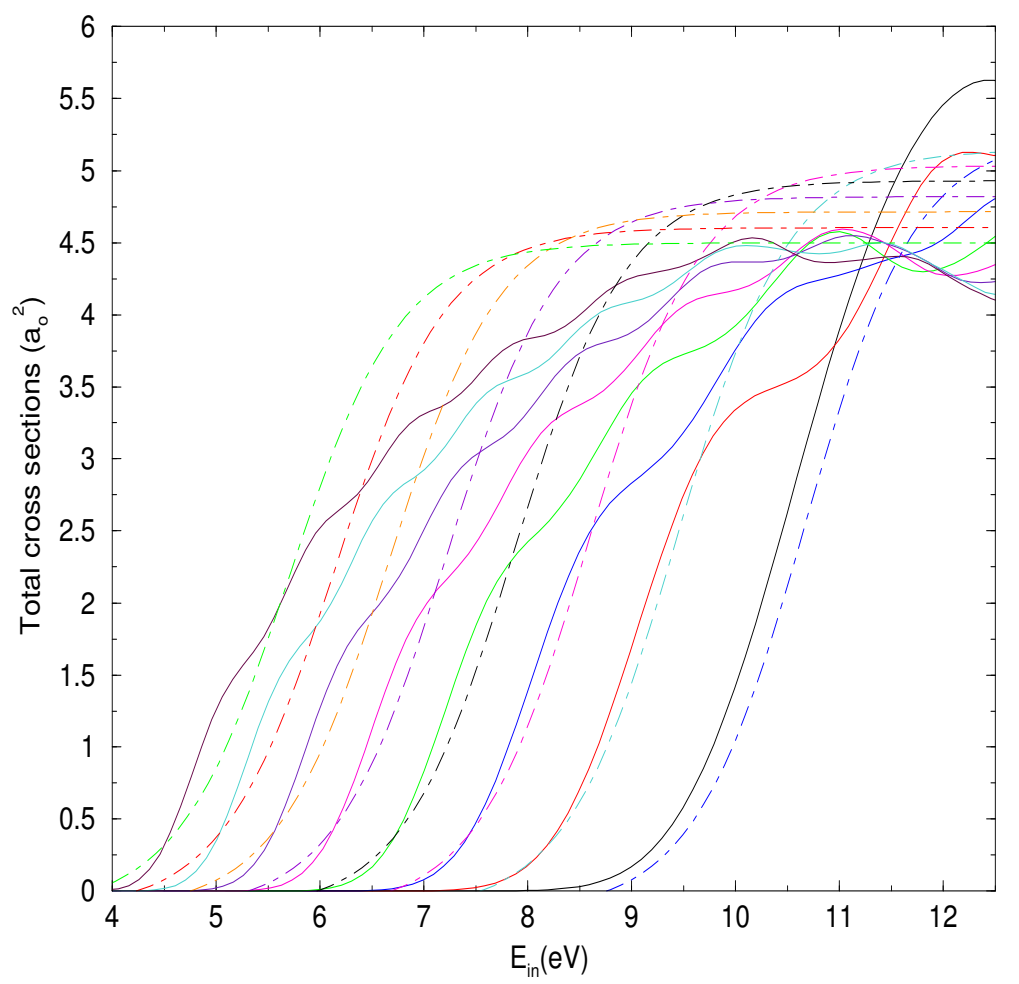

Figure 6. Electron impact dissociation cross sections for $T_{2}$ as a function of the incoming electron energy, $E_{i n}$ and initial vibrational level $v$. Full curves from right to left are the cross sections obtained from our full T-matrices calculations. Chained curves from right to left illustrate the curves obtained by applying our scaling law to each of the vibrational states described. 


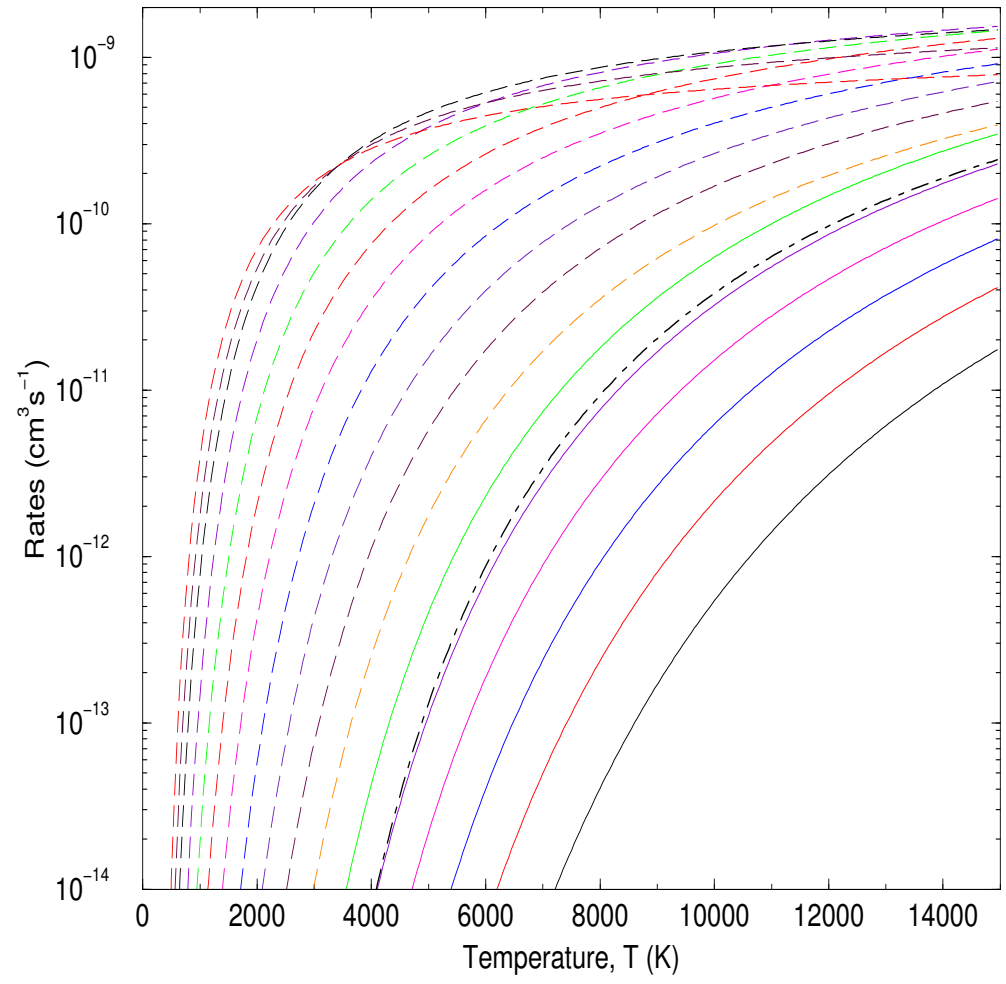

Figure 7. Electron impact dissociation rates as a function of initial HD vibrational state $v$. Full curves from the bottom are the rates for $v=0-5$, obtained from our full T-matrices calculations. Broken curves from the bottom are the rates for $v=6-16$. The chain curve represents the LTE rate. 


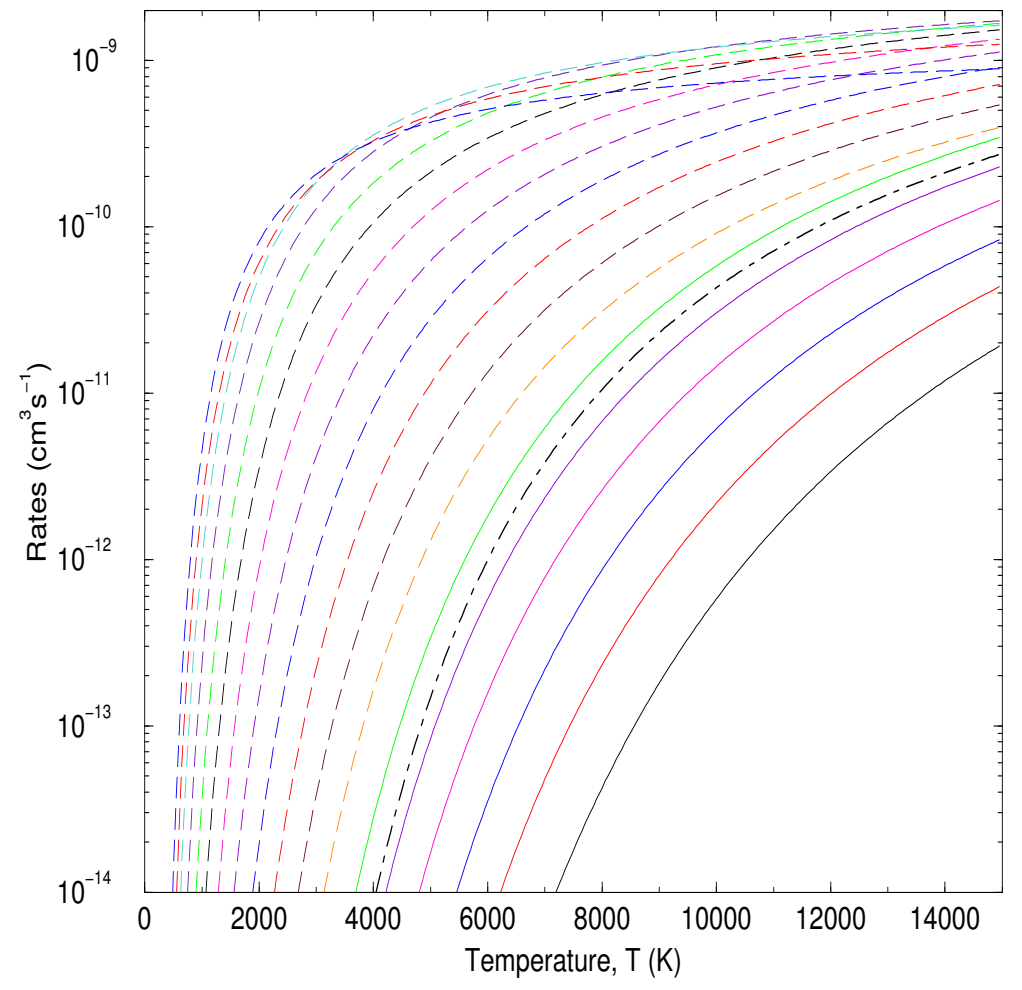

Figure 8. Electron impact dissociation rates as a function of initial HT vibrational state $v$. Full curves from the bottom are the rates for $v=0-5$, obtained from our full T-matrices calculations. Broken curves from the bottom are the rates for $v=6-17$. The chain curve represents the LTE rate. 


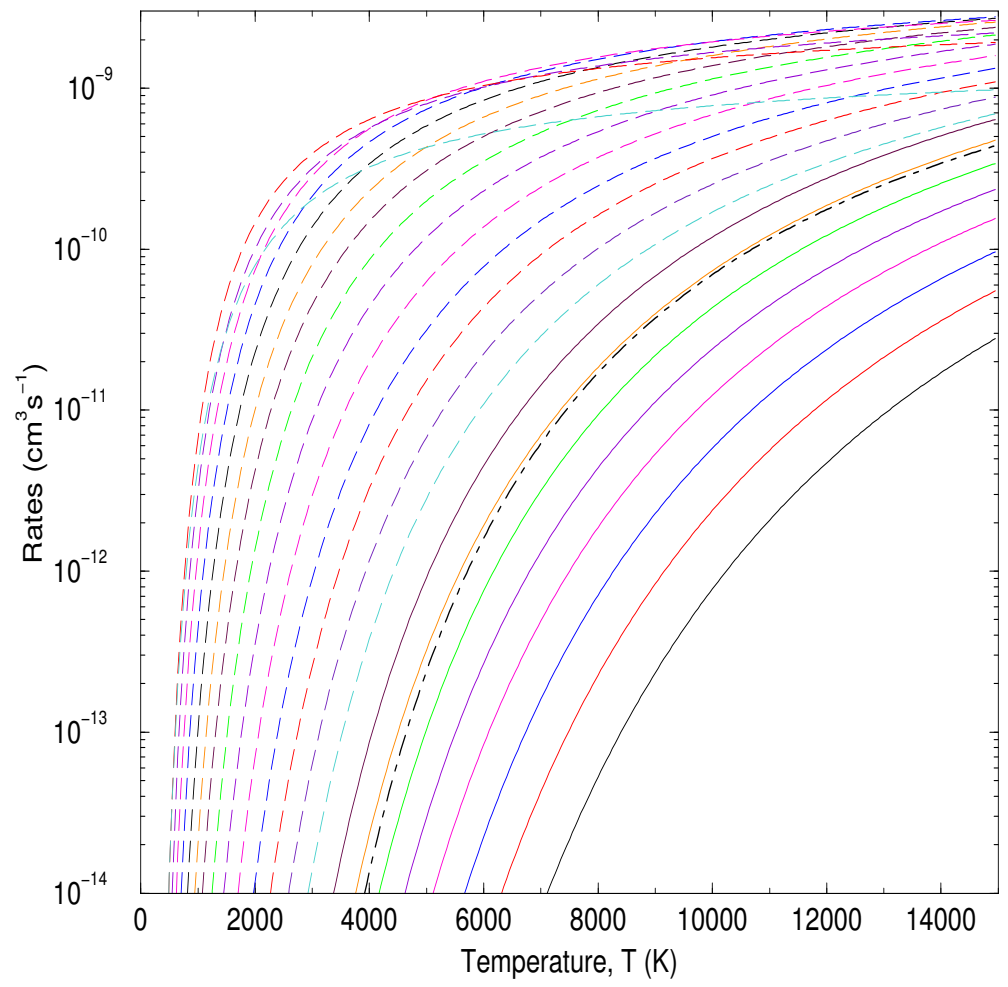

Figure 9. Electron impact dissociation rates as a function of initial DT vibrational state $v$. Full curves from the bottom are the rates for $v=0-7$, obtained from our full T-matrices calculations. Broken curves from the bottom are the rates for $v=8-22$. The chain curve represents the LTE rate. 


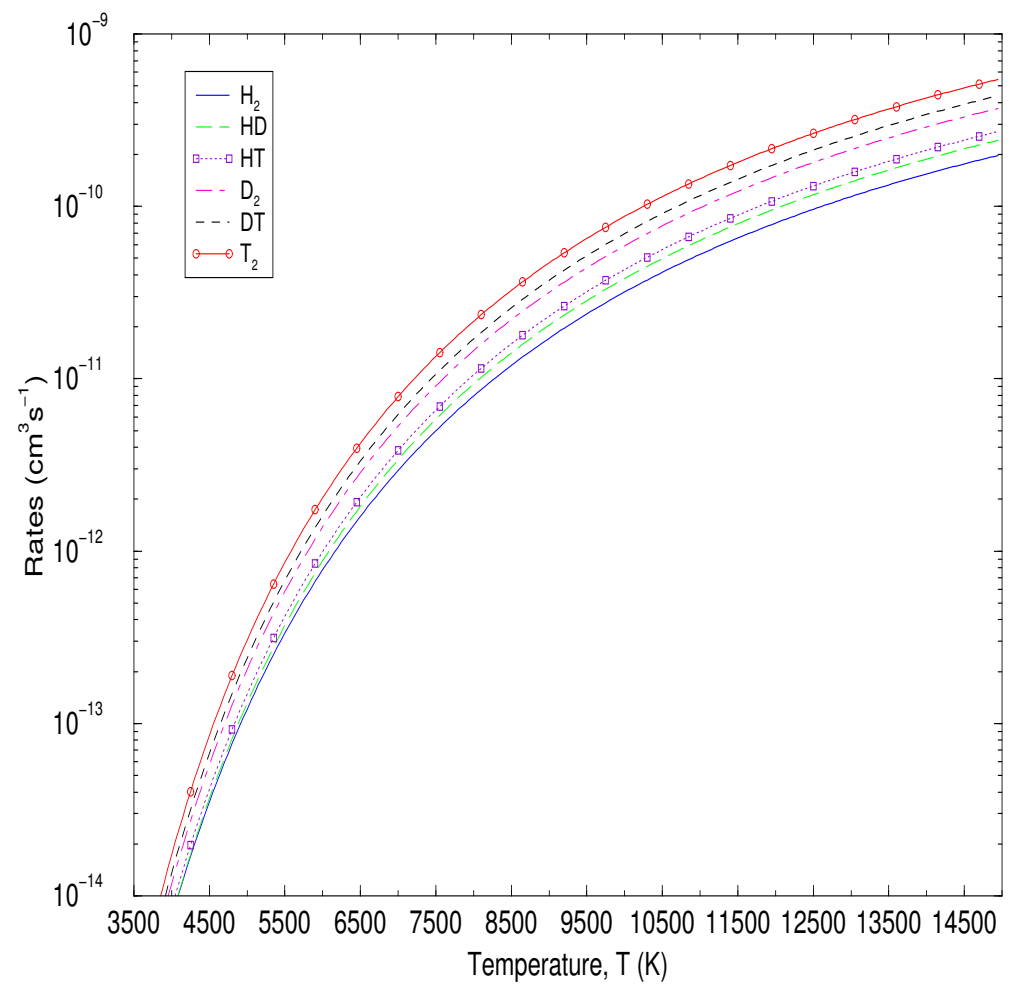

Figure 10. Comparison between the LTE rates of the different isotopomers studied. The rates increase with the reduced mass of the diatom. 